\title{
Uma definição ontológica de elementos de contexto relevantes na adaptação de documentos em ambientes hospitalares pervasivos
}

\author{
Vinícius Maran ${ }^{1}$ \\ Iara Augustin ${ }^{2}$ \\ Giovani Rubert Librelotto ${ }^{2}$ \\ Deise de Brum Saccol ${ }^{2}$
}

\begin{abstract}
Resumo: Um dos maiores desafios para sistemas pervasivos de saúde é fornecer aos usuários informações personalizadas, de acordo com as necessidades de cada pessoa ou sistema. Para que isso aconteça, é necessário que exista uma descrição das informações de contexto do ambiente, de forma reconhecível por outros sistemas computacionais. Uma das formas de se atingir essa funcionalidade é através do uso de ontologias, cujo objetivo é descrever um domínio de forma simples e extensível. Este artigo descreve o processo de construção de uma ontologia para a descrição de elementos de contexto relevantes em ambientes hospitalares pervasivos, com vistas à personalização de documentos no ambiente pervasivo prototipado na arquitetura de software ClinicSpace. Através da definição dessa ontologia, foi possível utilizar regras para a adaptação de documentos e restringir o acesso a informações clínicas na arquitetura ClinicSpace.
\end{abstract}

Palavras-chave: Adaptação consciente do contexto. Adaptação de conteúdo. Computação pervasiva. Contexto. Ontologias. Representação de conhecimento.

\begin{abstract}
One of the biggest challenges for pervasive health systems is to provide users with tailored information according to the specific needs of each person or computer system. For that happen, there must be a description of the context information of the environment in a recognizable way by other computer systems. One of the most frequently used form to describe areas in known as ontology, which aims to describe a field in a simple and extensible way. This article describes the process of building an ontology for the description of relevant elements of context in a hospital environment for customizing the documents in ClinicSpace environment. Through the definition of this ontology, it was possible to use rules for the adaptation of documents and restrict access to clinical information in ClinicSpace architecture
\end{abstract}

Keywords: Context. E-health. Modeling. Ontology. Knowledge representation. Pervasive computing.

\section{Introdução}

A expressão Computação Ubíqua, descrita por Mark Weiser [1], define um espaço onde dispositivos digitais e computadores de diferentes tipos estão totalmente integrados aos usuários e inseridos no seu ambiente de forma transparente. A principal motivação da computação ubíqua é auxiliar o usuário na realização das suas atividades diárias, abstraindo as questões computacionais envolvidas.

Aplicações pervasivas são compostas de componentes interativos centrados no usuário-final, que deseja realizar suas tarefas com a mínima interferência de sistemas desconhecidos por ele ou que necessitem de treinamento específico para a utilização desses sistemas. Alguns dos componentes desses sistemas devem atuar em background, buscando alcançar a invisibilidade da computação para o usuário-final [2].

\footnotetext{
${ }^{1}$ Curso de Ciência da Computação, UNIJUÍ (Universidade Regional do Noroeste do Estado do Rio Grande do Sul), Campus Santa Rosa - BR 344 - Santa Rosa (RS) - Brasil

\{vinicius.maran@unijui.edu.br\}

${ }^{2}$ Programa de Pós-Graduação em Informática, UFSM (Universidade Federal de Santa Maria) - Avenida Roraima, 1000 Santa Maria (RS) - Brasil

\{august, librelotto, deisedinf.ufsm.br\}
}

http://dx.doi.org/10.5335/rbca. 2013.2586

Revista Brasileira de Computação Aplicada (ISSN 2176-6649), Passo Fundo, v. 5, n. 1, p. 26-41, abr. 201326 
Considerando a Computação Ubíqua, os sistemas de saúde do futuro utilizarão tecnologias que possuem como funcionalidade básica o constante monitoramento do ambiente e das pessoas que estão inseridas nele, provendo a adaptação de conteúdo. Dessa forma, estes sistemas podem ser descritos como ambientes inteligentes, reativos e pró-ativos [3]. Para que os sistemas de saúde pervasivos tenham sucesso na constante adaptação de aplicações e documentos conforme as necessidades dos usuários e do ambiente (context-aware adaptation), é necessária uma modelagem eficiente do contexto, pois a captação e utilização dos elementos de contexto são essenciais para ambientes ubíquos [4].

Com vistas a experimentar soluções pervasivas no ambiente clínico, está em fase de prototipação uma ferramenta, desenvolvida na forma de arquitetura composta de serviços, chamada ClinicSpace [3], a qual visa adaptar o software de informação em saúde ao clínico que o utiliza (personalização e centralização no usuáriofinal).

Neste artigo, especificamente, descreve-se o processo de construção e a realização de testes em uma ontologia projetada para definir os principais elementos de contexto e conteúdo clínico que possam interferir na adaptação de documentos e exames hospitalares, sob o ponto de vista de profissionais clínicos. Com a definição dessa ontologia, foi possível definir regras para a adaptação de informações clínicas na arquitetura ClinicSpace de acordo com informações de contexto.

Na sequência, o artigo apresenta (i) uma breve introdução ao ambiente de computação pervasiva na saúde (Seção 2), a arquitetura ClinicSpace (Seção 3) e a área de ontologias como forma de representação de contexto (Seção 4), e (ii) o processo de definição de uma ontologia definida para representar os elementos de contexto relevantes na adaptação de conteúdo em hospitais pervasivos (Seção 5), a realização de testes na ontologia criada (Seção 6) e uma comparação com trabalhos relacionados (Seção 7). A Seção 8 descreve as conclusões do trabalho.

\section{Computação pervasiva e o hospital do futuro}

Em um ambiente pervasivo, dispositivos móveis e fixos trabalham de forma cooperativa e totalmente integrada ao ambiente, buscando auxiliar o usuário na execução das atividades realizadas nesse ambiente. Portanto, pode-se classificar o ambiente pervasivo como dinâmico e heterogêneo, já que atividades podem ser realizadas das mais diversas formas e há dispositivos de diversos tipos inseridos no ambiente [5] [6].

A necessidade da adaptação aos recursos se deve principalmente às trocas de contexto que ocorrem constantemente e devem ser capturadas pelos sistemas pervasivos. Tratar essa questão ainda é um desafio do ponto de vista computacional, pois as atividades humanas ainda são difíceis de serem previstas e modeladas de forma correta e passível de processamento [7]. Uma das áreas onde a computação pervasiva tem aparecido com relevância é a área de cuidados clínicos, na qual as pesquisas estão sendo conduzidas em duas linhas principais: (i) sistemas para ambientes hospitalares (healthcare); (ii) cuidados médicos para pacientes em suas próprias casas (homecare) [8].

A linha de pesquisa de sistemas hospitalares pervasivos tem como objetivo a construção de sistemas que auxiliem profissionais clínicos na realização de suas atividades hospitalares, da forma mais transparente possível [8]. Para realizar esse auxílio, os sistemas devem: (i) prever mudanças no ambiente (contexto), que ocorrem conforme as atividades são realizadas e (ii) prover uma adaptação constante dos sistemas, conforme se alteram os elementos de contexto que são monitorados [3]. Nesse escopo, o projeto ClinicSpace está sendo desenvolvido de forma a atender as exigências dos hospitais do futuro, permitindo com que médicos consigam modelar suas atividades (personalizar) e obter auxílio na realização das mesmas através da captura de contexto do ambiente hospitalar [3].

\section{O Projeto ClinicSpace}

O projeto ClinicSpace tem como foco principal prover auxílio a profissionais clínicos em suas tarefas diárias no ambiente hospitalar, utilizando tecnologias provenientes da área de Computação Pervasiva e Ubíqua. O sistema realiza o auxílio a profissionais com baixo grau de intrusão na realização de suas atividades diárias, já que se considera este um dos principais fatores de rejeição dos atuais sistemas hospitalares [4]. 
Para combater esse fator de rejeição, o sistema permite com que os médicos personalizem o sistema, modelando suas atividades diárias e o suporte computacional à sua execução [3]. A modelagem da arquitetura ClinicSpace é baseada na teoria da atividade [3] e no modelo apresentado por Ranganathan [9], no qual tarefas são definidas como "um conjunto de ações executadas colaborativamente por humanos e sistemas de computação pervasivos para atingir os objetivos" [9].

Tarefas são monitoradas por aplicações computacionais, as quais podem ou não interferir na sua execução de acordo com alterações nas informações de contexto. As tarefas podem ainda ser divididas em sub-tarefas, que se referem a pequenos serviços ou a outras tarefas que contêm, por sua vez, mais sub-tarefas associadas [10]. Na arquitetura ClinicSpace, tarefas são divididas em seis estados de execução possíveis (Figura 1).

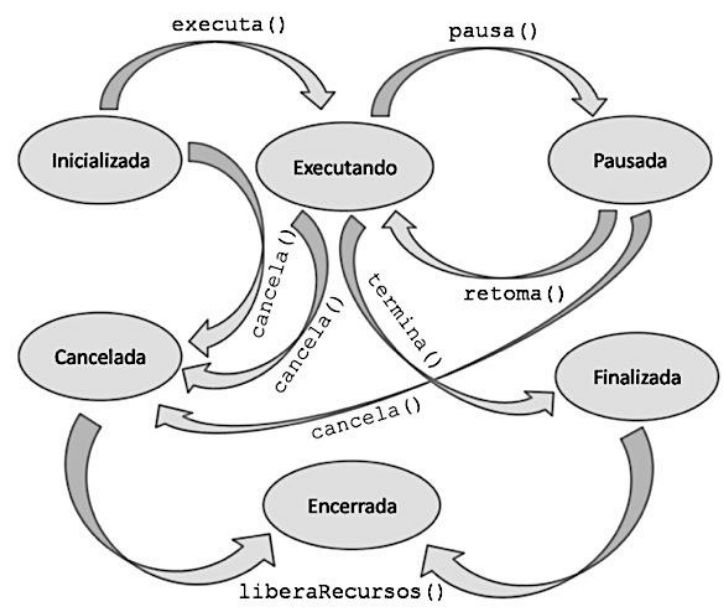

Figura 1: Diagrama de estados possíveis de uma tarefa [11]

O estado de cada tarefa define como esta será tratada pelo ambiente ClinicSpace, reservando recursos de execução ou liberando recursos quando estes não são mais necessários. As tarefas são subdivisões de uma atividade, que se refere a um processo realizado por pessoas (no caso da arquitetura ClinicSpace, os médicos). Quando a atividade é subdividida em tarefas, interferências computacionais podem ocorrer para auxiliar sua execução, tais como: (i) identificação automática dos pacientes, médicos e enfermeiros envolvidos na tarefa; (ii) a identificação do local onde a tarefa se realiza; e (iii) informações sobre a disponibilidade de exames laboratoriais do paciente.

\subsection{Utilização de informações de contexto}

O termo contexto pode ter diversas definições, pois se trata de um termo amplo e que abrange diversas áreas. Os elementos de contexto de algumas dessas áreas são facilmente identificados com a utilização de sensores, como, por exemplo, a temperatura do ambiente ou o tamanho do display de um dispositivo. Porém, outras formas de contexto ainda são complexas em relação ao reconhecimento e à modelagem, tal como a situação emocional de um usuário [12]. Para facilitar o entendimento sobre contexto, Chen \& Kotz [12] dividiram o tema em quatro conjuntos principais:

- Contexto de computação: Se refere às informações lógicas e físicas de dispositivos computacionais, sejam eles móveis ou fixos; tais como informações sobre capacidade de armazenamento ou informações sobre conectividade;

- Contexto de usuário: Se refere às informações baseadas no usuário em si, tais como perfil do usuário, atividades realizadas, localização atual do usuário, profissão, entre outras;

- Contexto físico: Se refere aos elementos físicos mensuráveis, que quase sempre são ambientes, tais como informações sobre iluminação, temperatura ou quantidade de pessoas presentes em um determinado local; 
- Contexto de tempo: Se refere às informações relacionadas ao tempo, que podem ser utilizadas para a consulta de um histórico de contextos para a tomada de decisões.

Outras propostas de representação de contexto, como por exemplo a de Brezzolin [13], propõem a separação de informações de contexto em: (i) contexto de sistemas, (ii) contexto de indivíduos, e (iii) contexto de projetos. Na arquitetura ClinicSpace, a definição de contexto foi baseada na definição proposta por Chen e Kotz [12] [3]. Dados de contexto podem ser representados de diversas formas. Strang e Popien [14] realizaram uma comparação entre as principais formas de representação de contexto e os requisitos que essas formas de representação atendem.

Esta comparação foi baseada em seis fatores, nomeados na tabela comparativa como composição distribuída $(c d)$; validação parcial $(v p)$; qualidade da informação (qi); incompleto e ambíguo (ia); nível de formalidade ( $n f o r)$, aplicabilidade em ambientes existentes $(a p l)$ e interoperabilidade entre linguagens de programação (int). Assim, constatou-se, na comparação, que a modelagem de contexto baseada em ontologias atende aos principais requisitos para a representação completa de contextos. O resultado do trabalho é apresentado na tabela a seguir (Tabela 1).

Tabela 1: Comparativo entre modelos de representação de contexto [14]

\begin{tabular}{c|ccccccc}
\hline Abordagens requisitadas & $c d$ & $v p$ & $q i$ & $i a$ & nfor & apl & int \\
\hline Chave valor & - & - & - & - & - & $*$ & $*$ \\
Esquema de marcação & $*$ & $*$ & - & - & $*$ & $*$ & $*$ \\
Orientado a objetos & $*$ & $*$ & $*$ & $*$ & $*$ & $*$ & - \\
Baseado em lógica & $*$ & - & - & - & $*$ & - & $*$ \\
Gráfico & - & - & $*$ & - & $*$ & $*$ & $*$ \\
Baseado em Ontologias & $*$ & $*$ & $*$ & $*$ & $*$ & $*$ & $*$ \\
\hline
\end{tabular}

Dessa forma, observa-se que a modelagem de contexto baseada em ontologias atende aos principais requisitos para a representação completa de contextos. Portanto, esse modelo foi o escolhido para a representação do domínio no qual o ambiente ClinicSpace se encontra.

\section{Ontologias e representação do conhecimento}

Ontologias possuem características e componentes comuns bem definidos. Os componentes básicos de uma ontologia são: i) classes - entidades organizadas taxonomicamente; ii) relações - o tipo de interação entre os conceitos de um domínio; iii) Axiomas - usados para modelar sentenças sempre verdadeiras; iv) instâncias utilizadas para representar elementos específicos, ou seja, os próprios dados [15]. De acordo com Guarino [16], uma ontologia pode ser definida como "uma teoria lógica para relacionar o significado pretendido de um vocabulário formal, isto é, seu comprometimento com uma conceitualização particular do mundo”.

Para a construção de ontologias é fundamental o uso de uma linguagem com semântica bem definida e expressiva para definir e criar relacionamentos entre os objetos. A linguagem OWL é uma das linguagens mais utilizadas para essa finalidade, sendo recomendada pela W3C (World Wide Web Consortium).

A OWL é uma linguagem para definição e instanciação de ontologias web. Provê uma forma de descrever as classes e as relações entre elas que são inerentes a documentos e aplicativos da Web [17]. Além disso, a linguagem permite verificar uma ontologia para determinar se sua lógica é consistente ou se há algum conceito falho. Uma ontologia OWL pode incluir: relações de taxonomia entre classes; propriedades dos tipos de dados e descrições dos atributos de elementos das classes, propriedades do objeto e descrições das relações entre elementos das classes, instâncias das classes e instâncias das propriedades. 
Todas as propriedades, classes e indivíduos são definidos com tags específicas, que definem o tipo do objeto na ontologia (se é uma propriedade, classe ou indivíduo), o nome único deste objeto na ontologia (conhecido como $\mathrm{URI}^{3}$ ) e propriedades que variam de acordo com o tipo de objeto definido.

Embora a linguagem OWL inclua um conjunto relativamente rico de construtores de classe, ela é muito fraca no que se refere às propriedades. Em particular, não há construtor de composição; por isso, é impossível capturar relacionamentos entre uma propriedade composta e outra propriedade (possivelmente composta). Para contornar esse problema, pode-se utilizar a linguagem SWRL [17], uma extensão da OWL para a construção de regras em uma representação OWL.

Essas regras permitem com que a estrutura da ontologia seja modificada com o tempo, através da utilização de um motor de inferência (reasoner), que realiza operações e pode mudar a estrutura, as informações e o significado das informações definidas em uma ontologia [17].

A SWRL é uma linguagem para escrever regras em lógica de primeira ordem. Utilizada em conjunto com OWL, forma uma dupla potente de linguagens de representação do conhecimento [17]. SWRL e OWL juntas permitem guardar informação categorizada e recuperá-la. Além disso, também permitem que um motor de inferência possa usar essa base para acrescentar novos conhecimentos à própria base, ou seja, as regras podem ser aplicadas sobre uma representação de conhecimento em OWL para representar um novo conhecimento.

SWRL e OWL juntas permitem guardar informação categorizada e recuperá-la. Além disso, também permitem que um motor de inferência possa usar essa base para acrescentar novos conhecimentos à própria base, ou seja, as regras podem ser aplicadas sobre uma representação de conhecimento em OWL para representar um novo conhecimento [17].

\section{Projeto de uma ontologia para a representação de elementos de contexto e informações clínicas de hospitais pervasivos}

Para iniciar o desenvolvimento da ontologia proposta, criou-se uma descrição formal representando o domínio que deve ser definido. A descrição relata as atividades cotidianas do ambiente hospitalar e os elementos de contexto presentes no ambiente e, posteriormente, levados em consideração na adaptação de documentos e conteúdo de forma geral no ambiente ClinicSpace.

Para definir a ontologia, seguiu-se o processo denominado Ontology Development 101 [22]. Esse processo tradicional consiste de um guia, com um conjunto de passos e dicas para a determinação de entidades e relacionamentos entre estas entidades, para a construção de uma ontologia. A Figura 2 apresenta o fluxo de trabalho definido no método Ontology Development 101, sendo que: (a) descreve os passos sugeridos pelo guia e (b) descreve um exemplo da concatenação dos passos, no processo de construção de uma ontologia.

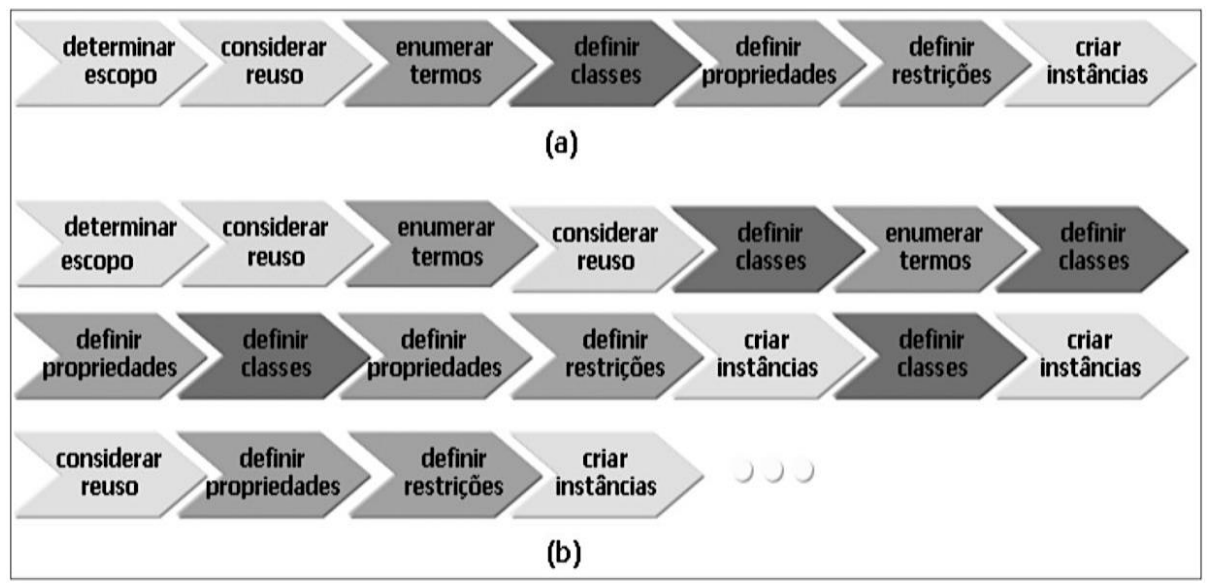

Figura 2: Processo de desenvolvimento proposto pelo guia Ontology Development 101 [22].

\footnotetext{
${ }^{3}$ URI (Uniform Resource Identifier) - Cadeia de caracteres compacta usada para identificar ou denominar um recurso na internet [17].
} 
A utilização dessa metodologia para o desenvolvimento da ontologia se deve principalmente ao equilíbrio entre as representações, utilizando restrições e a possibilidade de extensão da ontologia, permitindo futuras definições [22]. O escopo da ontologia proposta é restrito a informações de contexto relevantes no ambiente hospitalar pervasivo, no qual a arquitetura ClinicSpace está inserida e a representação de informações clínicas utilizadas pela arquitetura ClinicSpace.

Após a definição do escopo, consideramos o reuso de outras ontologias já definidas para a representação de contexto [19] [20] [21]. Porém, observou-se que essas ontologias destacavam informações de contexto de uma forma generalizada e que as mesmas não descreviam elementos de contexto específicos de ambientes hospitalares. Dessa forma, optou-se pela criação de representações de contexto. A próxima etapa na definição da ontologia proposta foi elencar os principais termos do domínio e representá-los na ontologia através de classes, propriedades e indivíduos. Esse processo é descrito a seguir.

\subsection{Representação de elementos de contexto}

A descrição dos elementos de contexto que devem ser representados na ontologia são limitados a agentes presentes no ambiente hospitalar (como por exemplo usuários, médicos e profissionais clínicos) e variáveis do ambiente hospitalar pervasivo que podem, de alguma forma, interferir na adaptação de documentos. Isso permite aos profissionais de saúde realizarem suas atividades cotidianas com o auxílio das tecnologias de computação pervasiva, e monitorarem o ambiente buscando por informações de contexto que possam interferir no andamento de uma atividade que vai ser iniciada ou que já está sendo executada.

As informações de contexto contidas neste ambiente podem ser classificadas de diferentes formas, tanto na forma física, através de informações de temperatura dos pacientes e localização dos profissionais de saúde, dispositivos, pacientes ou parentes dos pacientes dentro do ambiente hospitalar, quanto na forma lógica, detectando alterações em sistemas [4].

$\mathrm{O}$ acesso às informações pode ser realizado por pessoas que utilizam dispositivos dos mais diversos tipos, sejam eles móveis ou fixos. Dessa forma, as informações e interfaces devem se adaptar à heterogeneidade de dispositivos e componentes que compõem esses dispositivos. Médicos montam o modelo das suas atividades em seus dispositivos móveis e, a partir disso, o ambiente pervasivo monitora o ambiente de forma a auxiliar o profissional na realização das suas atividades cotidianas [3]. As atividades podem ser realizadas por um único profissional ou por um grupo de profissionais, que podem ser especializados em diferentes áreas médicas. Além disso, as informações relevantes são diferentes para cada especialidade médica ou profissional [4].

Apesar de os sistemas de informação em saúde possuírem todas as informações dos pacientes, devem ser repassadas aos profissionais somente as informações relevantes para a execução de suas atividades, ou as informações permitidas para o acompanhamento do estado de saúde de um paciente por seus parentes. A falta de garantia à confidencialidade é um dos problemas encontrados nos sistemas de saúde utilizados atualmente [3].

Para representar a permissão de acesso a informações clínicas por pessoas (indivíduos representados na ontologia), definimos a propriedade hasAccess, que, quando interligada entre um indivíduo da classe Person e um indivíduo da classe Content, representa a permissão de acesso da pessoa ao conteúdo clínico.

A partir de um estudo realizado por Laerum e Fazvaag [23], Silva [10] elencou 11 tarefas básicas que são realizadas por médicos com maior frequência em ambientes hospitalares, elas são apresentadas na Tabela 2 . Foram definidas as principais entidades de contexto envolvidas na realização destas tarefas. Essas entidades foram representadas na ontologia (Seção 5.3).

Tabela 2: Atividades mais comuns em ambientes hospitalares [10]

\begin{tabular}{cc} 
ID & Descrição \\
\hline 1 & Revisar os problemas do paciente \\
2 & Procurar informações específicas no registro do paciente \\
3 & Obter os resultados de novos testes e investigações \\
4 & Adicionar notas diárias sobre condições do paciente \\
5 & Requisitar análises laboratoriais \\
6 & Requisitar exames de video/imagem \\
7 & Obter resultados laboratoriais \\
8 & Obter resultados de exames de video/imagem \\
9 & Requisitar tratamentos \\
10 & Escrever prescrições \\
11 & Registro de códigos para diagnóstico e procedimentos executados \\
\hline
\end{tabular}

Revista Brasileira de Computação Aplicada (ISSN 2176-6649), Passo Fundo, v. 5, n. 1, p. 26-41, abr. 201331 
A partir do momento que se cria a descrição do domínio que a ontologia deve representar, pode-se começar a definir as principais classes da ontologia, que representam informações de contexto e informações sobre as atividades realizadas, no ambiente hospitalar pervasivo com o objetivo de auxiliar no processo de adaptação de documentos e exames no ambiente ClinicSpace.

\subsection{Representação de informações clínicas}

Para realizar a modelagem de documentos clínicos em uma ontologia para a utilização pelo ClinicSpace, foram utilizadas informações de documentos e exames clínicos provenientes de instituições hospitalares. A partir de uma lista de documentos utilizados com maior frequência em atividades hospitalares por profissionais clínicos, obtida através de uma pesquisa realizada em um hospital no interior do Rio Grande do Sul (Tabela 3), determinou-se o conhecimento clínico envolvido e as informações de contexto relacionadas no preenchimento desses documentos e modelou-se essas informações na ontologia.

Tabela 3: Lista de documentos frequentemente utilizados no ambiente hospitalar

\begin{tabular}{ll}
\hline Número & Documentos utilizados \\
\hline $\mathbf{1}$ & Relatório de exame laboratorial \\
$\mathbf{3}$ & Relatório de evolução de enfermagem \\
$\mathbf{4}$ & Prontuário médico do paciente \\
$\mathbf{5}$ & Relatório cirúrgico \\
$\mathbf{6}$ & Prescrição médica diária \\
$\mathbf{7}$ & Relatório de anamnese diária \\
$\mathbf{8}$ & Relatório de evolução diária \\
$\mathbf{9}$ & Registro de internação do paciente \\
$\mathbf{1 0}$ & Solicitação de exame \\
$\mathbf{1 1}$ & Solicitação de encaminhamento para outro instituto hospitalar \\
\hline
\end{tabular}

Após a listagem de quais documentos eram mais utilizados em atividades clínicas por médicos, utilizouse os modelos de documentos usados em instituições hospitalares para a extração de informações clínicas relevantes. Um exemplo de mapeamento de informações de um documento para uma representação ontológica é apresentado na Figura 3. 


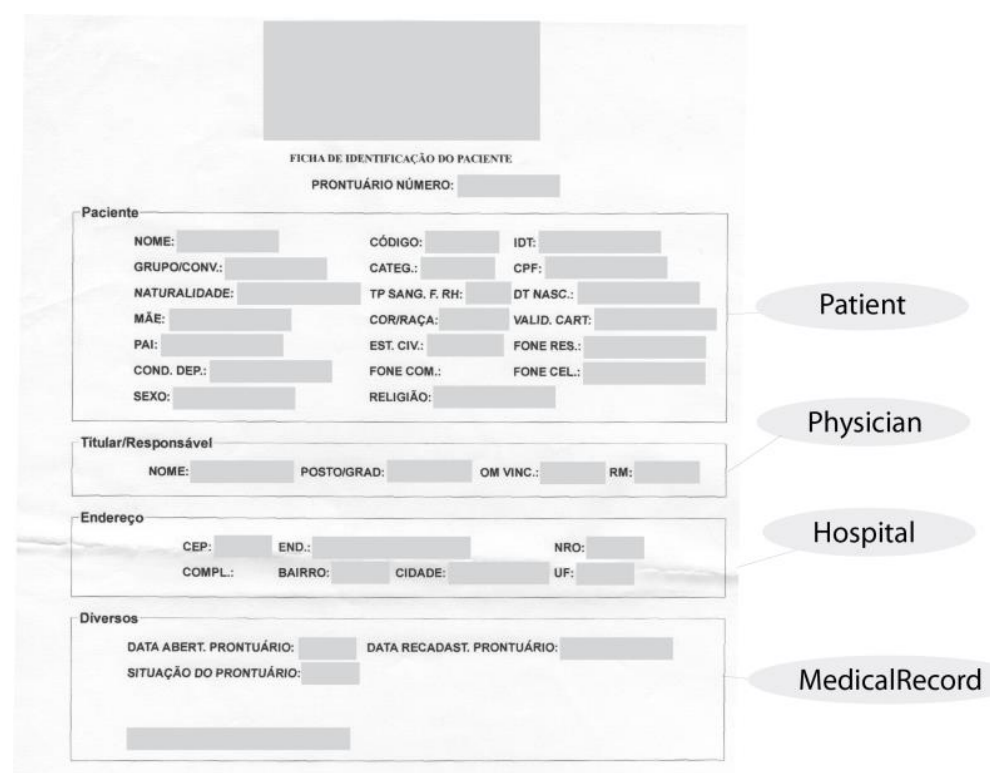

Figura 3: Exemplo de representação das informações clínicas de um documento clínico

As informações foram omitidas na Figura 4 por questões de ética em relação às informações reais de pacientes e foram modeladas através de DataProperties ${ }^{4}$ de classes na ontologia. Além disso, foram modeladas as ligações entre as classes.

A modelagem dos dados clínicos na ontologia foi realizada tendo como base duas superclasses principais: (i) a classe DocumentPart, que define uma pequena parte de um documento, suas informações e os elementos de contexto que permitem a adaptação desse documento (por exemplo pront_paciente, pront_titular e pront_diversos, na anterior) e (ii) a classe Document, que define um conjunto de partes de documentos e associa essas partes de documentos a elementos de contexto relevantes.

Essa abordagem foi escolhida considerando as condições de uso. Por exemplo, se um exame de sangue padrão é realizado por um determinado paciente, este pode conter informações de interesse de diferentes especialidades médicas. Então, o serviço de seleção contextualizada pode selecionar apenas partes ou versões deste documento, conforme a necessidade da consulta de uma determinada especialidade.

\subsection{Modelagem das principais classes}

Após o levantamento de informações de contexto e informações clínicas, foram definidas as principais classes da ontologia, também chamadas de superclasses, pois definem as principais áreas de contexto que foram sendo refinadas durante a modelagem da ontologia. Elas representam elementos de contexto que podem influenciar o ambiente pervasivo na adaptação de conteúdo.

Para realizar a modelagem da ontologia, utilizou-se o software Protégé na versão 3.4.4 [24]. As principais superclasses da ontologia construída são apresentadas na Figura 4.

\footnotetext{
${ }^{4}$ Relacionamento entre um indivíduo da ontologia e um tipo de dado definido na ontologia [17].
} 


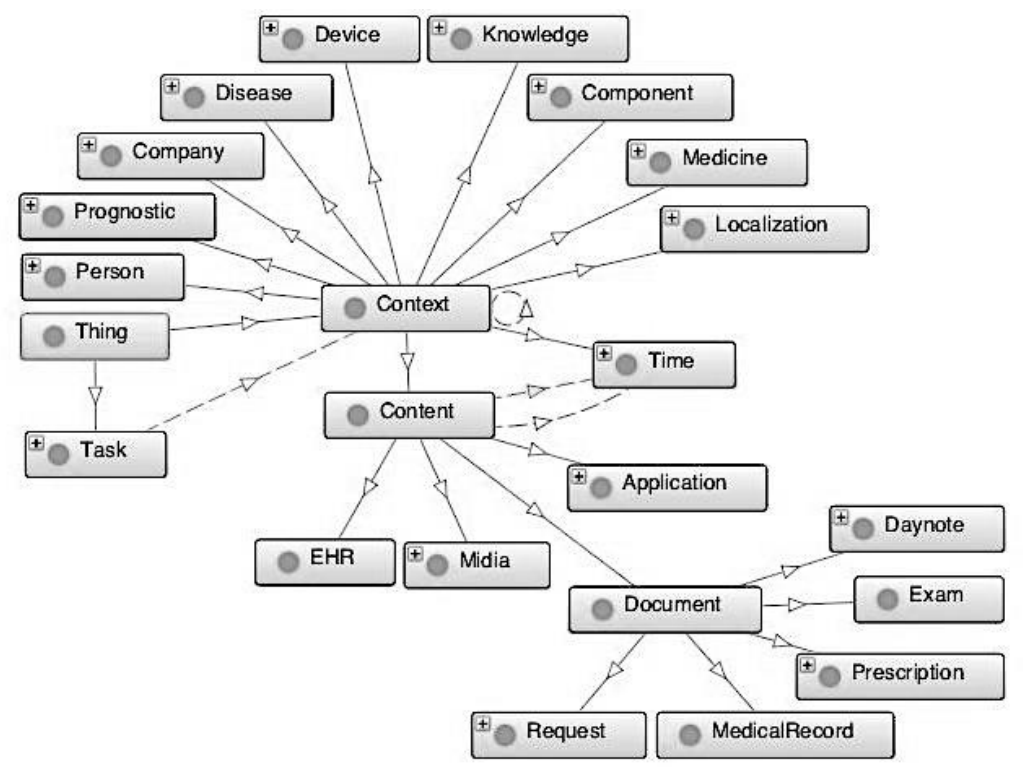

Figura 4: Principais classes da ontologia proposta

A partir da pesquisa realizada no domínio hospitalar e as atividades realizadas nesse ambiente, foram definidas as principais classes da ontologia, também chamadas de superclasses, que definem as áreas de contexto abordadas na arquitetura ClinicSpace. Essas áreas foram sendo refinadas durante a modelagem da ontologia, elas representam elementos de contexto que podem influenciar o ambiente pervasivo, na seleção contextualizada de conteúdo e adaptação em relação às funcionalidades. Como principais elementos de contexto, definiu-se:

- Aplicação (Application) - As aplicações descritas são os próprios serviços e subsistemas da arquitetura ClinicSpace, ou sistemas externos que desejam ter acesso às informações utilizadas pelo ClinicSpace;

- Tempo (Time) - O tempo é determinante na realização de tarefas, pois de acordo com a duração das atividades, o sistema pode distribuir tarefas entre profissionais clínicos, dependendo se o profissional está perto do horário de saída ou está no seu turno de trabalho normal, por exemplo;

- Doença (Disease) - As doenças são relacionadas com informações presentes nos documentos relacionados aos pacientes. Além disso, quando um paciente é detectado pelo sistema e não foi atendido ainda, pode receber um atendimento prioritário, caso o sistema verifique que este paciente possua uma doença séria ligada com a sua ficha hospitalar (há a possibilidade de estender essa classe a outras definições já consolidadas);

- Localização (Localization) - A localização dos recursos e pessoas é determinante na delegação de tarefas, recursos e no acesso às informações clínicas. O sistema filtra o acesso às informações de pacientes somente quando os médicos estão presentes no ambiente hospitalar, mesmo que eles possuam acesso às interfaces do ClinicSpace em seus dispositivos móveis pessoais. Em relação à delegação de tarefas e recursos, o sistema delega tarefas e realiza avisos de acordo com a localização de recursos, pacientes e médicos. Por exemplo, se um médico está realizando um procedimento na unidade de terapia intensiva, este profissional não pode ser interrompido pelo sistema ou receber avisos externos não relacionados com a atividade que ele está realizando no momento, eis que de alto risco;

- Pessoa (Person) - Todas as atividades da arquitetura ClinicSpace são realizadas baseando-se nas tarefas realizadas por pessoas e elementos de contexto do ambiente. Dessa forma, torna-se indispensável a representação das pessoas presentes no ambiente hospitalar. Tanto em relação ao paciente, quanto em relação aos funcionários e profissionais clínicos envolvidos nas atividades médicas; 
- Medicamento (Medicine) - De acordo com as necessidades do paciente e dos pedidos realizados pelo médico, o sistema pode encaminhar o paciente para o setor responsável pelos medicamentos ou emitir uma receita médica para o paciente. Além disso, o sistema analisa o histórico de medicamentos do paciente, se este está disponível e informa ao médico sobre esse histórico no momento da consulta (há a possibilidade de estender essa classe a outras definições já consolidadas;

- Componente (Component) - Os componentes são determinantes na coleta e apresentação de informações. Basicamente, todos os dispositivos são compostos de componentes, os quais determinam as características e capacidades de um dispositivo em si;

- Material para exame (MaterialForClinicExamination) - Caso um paciente precise realizar um exame, ele pode ser encaminhado ao setor responsável de acordo com a urgência, ou seja, se o exame precisa ser realizado o mais rápido possível, o paciente é encaminhado ao laboratório do próprio hospital;

- Conhecimento (Knowledge) - O conhecimento médico define o acesso aos documentos de pacientes pelos profissionais clínicos. Por exemplo, se um especialista em pediatria está presente na instituição hospitalar, ele terá acesso aos documentos relacionados à área médica de pediatria no atendimento aos seus pacientes. Além disso, em caso de emergência e dependendo da disponibilidade do médico, ele pode receber a delegação de atendimento a um paciente que tenha algum problema relacionado à sua especialidade médica (há a possibilidade de estender essa classe a outras definições já consolidadas);

- Prognóstico (Prognostic) - O prognóstico do paciente pode determinar a delegação de tarefas a determinados profissionais clínicos. Por exemplo, se o paciente está esperando o atendimento de um médico e apresenta o prognóstico "vômito", o sistema dispara um evento pedindo a colaboração de um profissional clínico para fazer um atendimento ao paciente;

- Dispositivo (Device) - Os dispositivos utilizados no ambiente hospitalar definem a forma como a informação será apresentada, a formatação da informação é de acordo com as características da rede e do dispositivo, tais como largura de banda, capacidade de armazenamento e propriedades de um display, caso o dispositivo o possua. Dispositivos podem ser fixos - computadores e terminais de salas específicas, ou móveis - telefones celulares ou sensores.

Além das classes mostradas anteriormente, foram definidas outras subclasses especificando cada um dos elementos de contexto, e permitindo que mais classes sejam definidas por sistemas ou utilizadores da ontologia, de acordo com as suas necessidades. Portanto, a ontologia construída provê recursos de extensão para a sua utilização em outros sistemas hospitalares pervasivos. Além disso, foram definidas classes e propriedades que representam as informações clínicas em documentos que são frequentemente utilizados em sistemas hospitalares.

Dessa forma, as informações contidas nos documentos clínicos possuem semântica e podem ser facilmente convertidas para outros formatos, como por exemplo os padrões CCR [25] e HL7 [26], utilizados em diversos sistemas que utilizam prontuário eletrônico. A Figura 5 apresenta uma representação ontológica de documentos clínicos. 


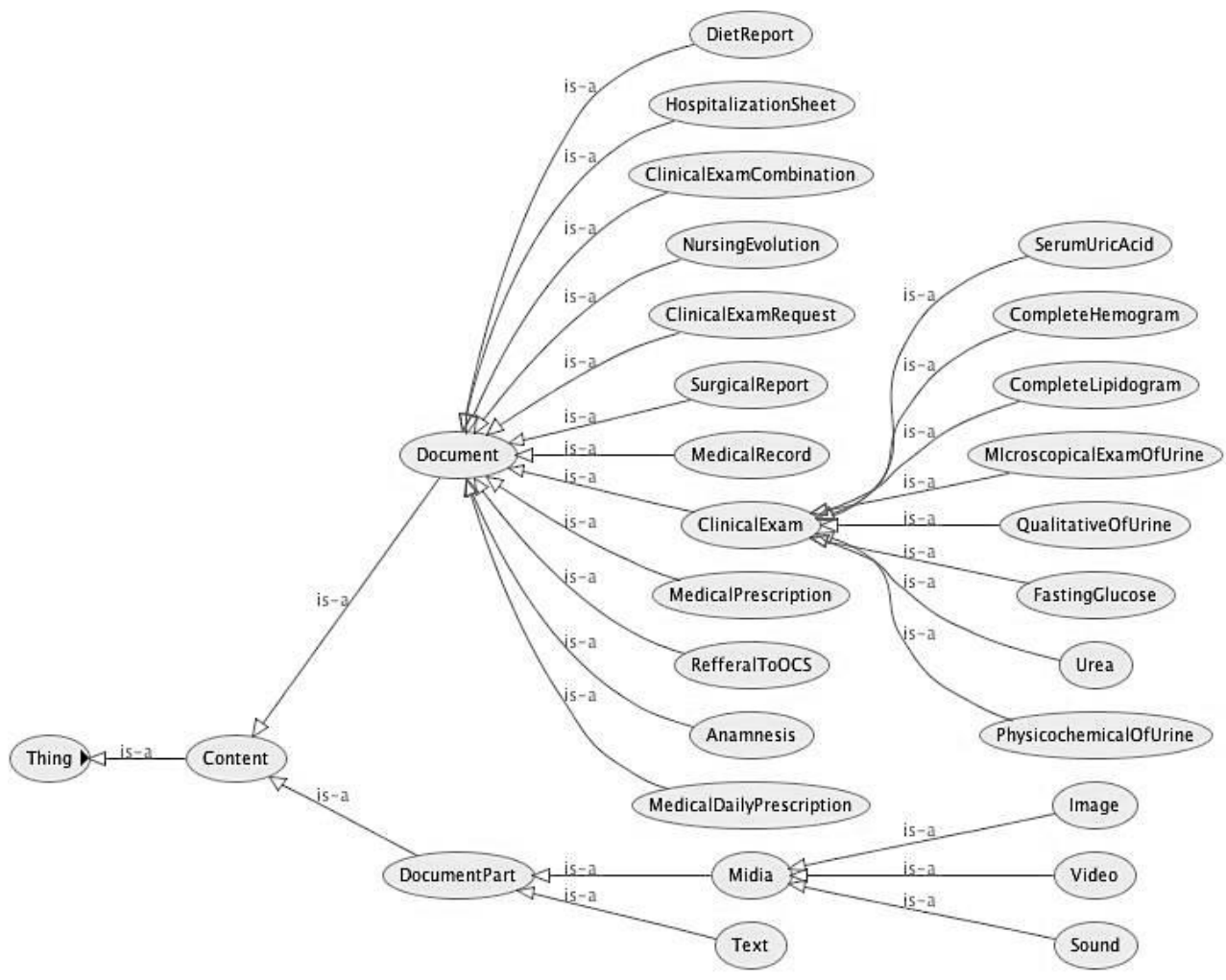

Figura 5: Modelagem de documentos clínicos na ontologia.

Para validar os termos representados na ontologia e a integração entre estes, realizou-se algumas consultas e inferências para demonstrar o uso da ontologia construída em situações de uso comuns na arquitetura ClinicSpace.

\section{Testes e inferências}

Para a realização de consultas, utilizou-se a linguagem SQWRL [27] e a ferramenta Protégé. Inicialmente, definiu-se um conjunto de consultas e regras de inferência baseadas em um estudo realizado em parceria com um hospital universitário do Rio Grande do Sul, onde foram elencadas as tarefas e consultas mais comuns realizadas em sistemas hospitalares.

Dessa forma, as definições propostas foram validadas com profissionais do domínio descrito pela ontologia. Após a definição das consultas e regras, indivíduos de classes variadas foram inseridos na ontologia desenvolvida. Esses indivíduos possuem relações entre si que foram descritas utilizando propriedades.

\subsection{Testes de consulta e inferência}

Em uma primeira etapa de testes, realizaram-se consultas simples na ontologia, buscando obter informações sobre um determinado grupo de indivíduos. A seguir, são apresentadas as consultas realizadas na ontologia para testes, o código SQWRL referente às consultas realizadas e os resultados nestas obtidos.

- Consulta 1. Deseja-se saber o número de pacientes adultos que estão presentes no hospital no momento da consulta (considerando que pacientes adultos possuem mais de 17 anos). O trecho de código SQWRL escrito para realizar esta consulta na ontologia é apresentado no trecho de código a seguir.

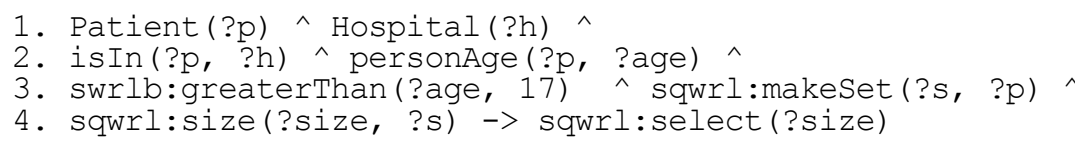


Como resultado, a consulta retornou o número de pacientes que estavam no hospital e que tinham a idade maior que 17 anos. A Consulta 2 representa um exemplo de consulta baseada em um indivíduo específico da ontologia, consultando dados e indivíduos que possuam alguma ligação específica com esse indivíduo.

- Consulta 2. Deseja-se obter os nomes dos pacientes que estão sendo tratados atualmente pelo indivíduo da classe Doctor com nome "Doutor_Carlos". O trecho de código SQWRL escrito para realizar essa consulta na ontologia (a) e o resultado apresentado pelo software Protégé (b) são apresentados na Figura 6.

(a)

1. Patient(?p) ^ Doctor(Doutor Carlos) ^

2. treatedBy(?p, Doutor Carlos) $\wedge$

3. personName (?p, ?name) $\rightarrow$

4. sqwrl: select (?name)

Figura 6: Consulta 2 (b)

\begin{tabular}{|l|}
\hline ?name \\
\hline paciente dois \\
paciente tres \\
paciente um
\end{tabular}

Na consulta 3, testa-se a obtenção de diversos dados relacionados a indivíduos de uma mesma classe.

- Consulta 3. Deseja-se saber o número de identificação único (representado pelo campo $i d$ ), o nome e a idade dos pacientes que estão nas dependências do hospital. O trecho de código SQWRL escrito para realizar essa consulta na ontologia (a) e o resultado da consulta (b) são apresentados na Figura 7.

(a)

1. Patient (?p) ^ Hospital(?h)^

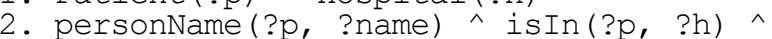

3. personAge (?p, ?age) ^ personID(?p, ?id)

4. $\rightarrow$ sqwrl:select(?id, ?name, ?age)

Figura 7: Resultados da consulta 3 (b)

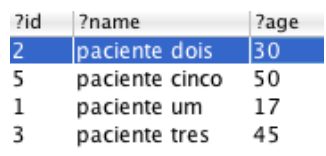

Apesar de proporcionar a obtenção de dados da ontologia, as consultas não contemplam a inserção ou modificação do conhecimento representado na ontologia. Dessa forma, torna-se necessária a utilização de inferências, que permitem a adição ou alteração de um conhecimento presente na ontologia. Para a realização de inferências sobre a ontologia proposta, foram elaboradas algumas constatações para testar se a ontologia, através de regras de inferência, era capaz de realizar a validação dessas constatações.

Em seguida, montou-se um conjunto de regras, referentes a essas constatações, utilizando a linguagem SWRL, e realizaram-se os testes de inferência, utilizando o software Protégé versão 3.4 .4 e o plugin Jess na versão $7.1[28]$.

- Inferência 1. Definiu-se uma regra que estabelece que todos os parentes de pacientes que estão no mesmo ambiente hospitalar que o paciente tem o direito de acessar as informações de diagnóstico desse paciente. $\mathrm{O}$ trecho de código apresentado a seguir descreve o código SWRL referente à regra definida nessa inferência.

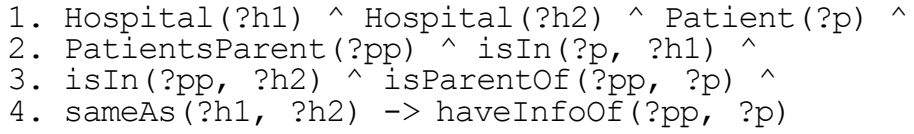

Como resultado para essa inferência, alterações foram realizadas na ontologia, criando a ligação haveInfo $O f$ entre os parentes de um paciente e o paciente, permitindo assim que esses parentes tenham permissão de acesso às informações sobre o paciente no ambiente hospitalar pelo sistema pervasivo.

- Inferência 2. Definiu-se uma regra que diz respeito ao tratamento diferenciado do sistema a uma pessoa que está no ambiente hospitalar e está sendo monitorada por um sensor. A partir dessa premissa, a pessoa passa a ser tratada como paciente pelo sistema hospitalar. $\mathrm{O}$ trecho de código apresentado na Figura 33 descreve o código SWRL referente à regra definida nesta inferência.

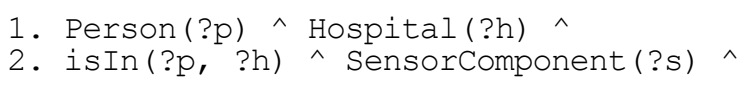


Após a inferência utilizando a regra apresentada, todas as pessoas que estavam no hospital e estavam sendo monitoradas por um sensor passaram a pertencer à classe Patient e, consequentemente, tratadas pelo sistema hospitalar como pacientes.

Com a realização dos testes de representação foi comprovado que a ontologia criada representa os principais elementos de contexto e informações clínicas frequentemente utilizadas por médicos. A próxima etapa de testes se refere a testes de utilização da ontologia. Esses testes são descritos na próxima seção.

\subsection{Testes de integração com a arquitetura ClinicSpace}

Realizaram-se também testes de uso, medindo o tempo de carregamento da ontologia, definida em um total de setenta classes, na utilização em um sistema desktop. Utilizou-se uma máquina com processador Intel E5500 2,8ghz, 2gb ddr2, HD de 500gb, Java SE v1.7.0, API Jena para a utilização da ontologia em conjunto com a linguagem Java e sistema operacional Windows 732 bits. Os serviços básicos da arquitetura ClinicSpace foram inicializados e, após, foram feitos os testes de carregamento da ontologia. Os tempos de carga da ontologia em relação ao número de indivíduos na ontologia são apresentados na Figura 8.
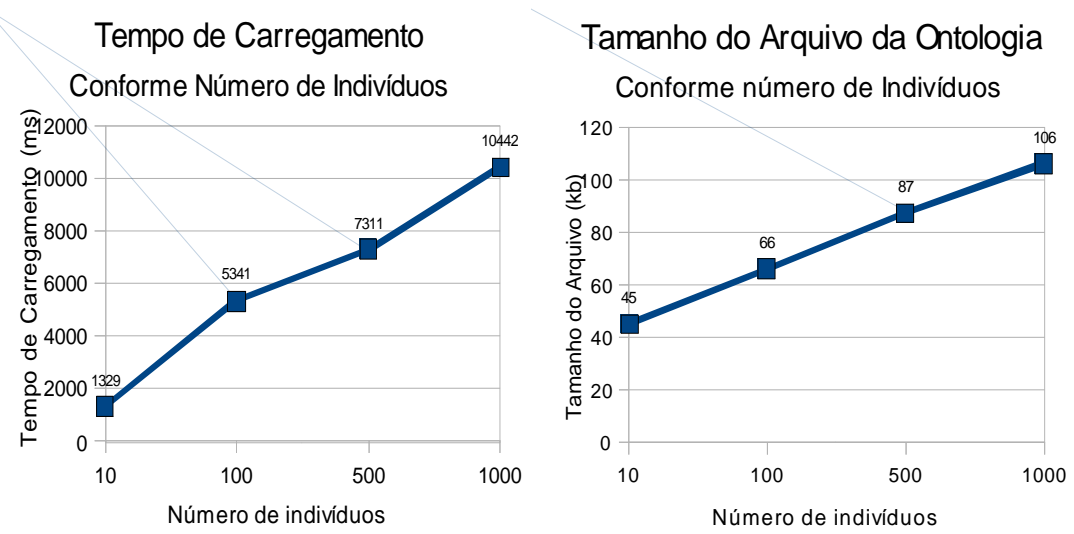

Figura 8: Resultados dos testes de carregamento da ontologia.

Como se pode observar, o número de indivíduos na ontologia interfere diretamente no tempo de carregamento da ontologia para o seu uso posterior em sistemas computacionais. Além disso, a inserção de dados referentes às propriedades desses indivíduos contribui para o aumento do tempo de carregamento.

Nesse teste, foram medidos apenas os tempos de carregamento da ontologia em memória realizado pela API Jena, desconsiderando o tempo de inicialização da arquitetura ClinicSpace. Como o aumento no tempo de carga da ontologia foi quase linear, de acordo com o número de indivíduos, observou-se que será necessária a integração de uma forma de persistência de ontologias (sugerida como trabalho futuro no Capítulo 8). Dessa forma, espera-se que o número de indivíduos não interfira de forma direta no tempo de carga das definições da ontologia na arquitetura.

\section{Trabalhos relacionados}

Segundo Bettinia [18], a variação de ontologias geralmente utilizada para a representação de contexto é a OWL-DL, uma vez que ela está se tornado um padrão para vários domínios de aplicação e é suportada por um grande número de servidores de raciocínio.

Essa variação de ontologias permite a modelagem de um domínio específico sem as limitações encontradas na variação OWL-Lite. Várias ontologias baseadas na linguagem OWL têm sido propostas para representar a descrição distribuída para os dados do contexto. As propostas mais promissoras são: 
- Chen et al [19]: define uma ontologia para modelagem de contexto em ambientes pervasivos de forma genérica e compatível com outras ontologias, porém essa ontologia não modela a integração entre contexto e informações de documentos envolvidos em atividades;

- Zhang et al [20] define uma ontologia para ambientes de casas inteligentes. Essa ontologia distribuída pode ser integrada com modelos específicos de aplicação de contexto por meio de extensões da linguagem OWL;

- Koay et al [21] define uma ontologia que modela requisitos não funcionais de sistemas de saúde pervasivos e propõe um conjunto de inferências para adaptação de recursos. Porém, não define a modelagem das tarefas clínicas e os documentos frequentemente utilizados nessas tarefas;

- Villalon et al [29] define uma ontologia que modela informações de contexto relevantes em ambientes móveis. O principal foco da definição da ontologia é a descrição de dispositivos e redes que compõe o ambiente de computação móvel. Além disso, a definição permitiu a interligação da mesma com outras ontologias.

Nos trabalhos pesquisados, a definição de elementos de contexto e informações de domínio não foram feitas na mesma ontologia. Essa característica dificulta a realização de ações nos dados de domínio de acordo com informações de contexto coletados por sistemas ubíquos, pois é necessária a definição e modificação de definições nas informações de domínio com base no contexto.

Dessa forma, a ontologia proposta neste trabalho utilizou a definição de elementos de contexto e informações de domínio de forma interligada, permitindo com que a arquitetura ClinicSpace adapte conteúdo clínico de acordo com informações de contexto.

\section{Conclusões}

A ontologia proposta tem como objetivo definir as principais classes de representação de um ambiente hospitalar pervasivo, de acordo com a visão da arquitetura ClinicSpace. A ontologia, de modo geral, facilita a descrição do contexto e a representação de um determinado domínio de conhecimento. Além disso, ela possibilita o reuso do conhecimento e das definições, facilita possíveis extensões, e permite o compartilhamento de informações entre diversos tipos de ambientes e dispositivos.

A aplicação de inferências e consultas sobre uma base de conhecimento possibilita a obtenção de informações importantes relativas ao contexto, gerando um novo conhecimento e viabiliza a adequação das informações e tarefas aos ambientes dinâmicos e heterogêneos envolvidos. Atendendo, assim, aos requisitos de um ambiente pervasivo.

Com a ontologia criada será possível estender as capacidades de adaptação da arquitetura ClinicSpace, possibilitando a utilização de informações de contexto no momento da execução de tarefas e a utilização de informações do histórico de contextos registrados no sistema através de inferências realizadas sobre essa ontologia.

O processo de criação de uma ontologia não é trivial, pois como se pode observar, durante a execução do trabalho, há alguns momentos onde erros de interpretação e ambiguidades podem aparecer. A ontologia apresentada servirá como base para a próxima etapa da pesquisa, que trata de modelar um serviço para a adaptação de conteúdo no ambiente ClinicSpace.

Além da criação do serviço de adaptação de documentos baseado em informações de contexto, como trabalhos futuros sugere-se:

- A criação de novas regras de inferência em SWRL e a criação de consultas em SQWRL que representem operações cotidianas e que precisam de adaptação de acordo com o contexto captado pela arquitetura ClinicSpace;

- A extensão da representação de conhecimento clínico na ontologia;

- A pesquisa de formas de persistência de contexto representado em ontologias e integração dessas formas de persistência com a arquitetura ClinicSpace. 


\section{Referências}

[1] Weiser, M. “The Computer of the 21st Century”, Em: Scientific American, volume 265, número 9, 1991.

[2] Kukhun, D.A.A., Sedes, F. "Step Towards Pervasive Software: Does Software Engineering Need Reengineering?" Livro Complex Systems Concurrent Engineering, Ed. Springer. ISBN 978-1-84628-975-0, 2007, pp. 143-150. 2007.

[3] Ferreira, G., Silva, F., Librelotto, G. R., Augustin, I. "Middleware for Management of End-User Programming of Clinical Activities in a Pervise Environment" Em: 4th International Conference on Communication System Software and Middleware. WMUPS 2009.

[4] Vicentini, C., Machado, A., Ferreira, G. L., Lorenzi, F., Augustin, I. "PEHS - Arquitetura de um Sistema de Informação Pervasivo para Auxílio às Atividades Clínicas" Em: Revista Brasileira de Computação Aplicada-RBCA. v. 2, n. 2. 2010.

[5] Maran, V., Machado, A., Saccol, D. B., Augustin, I. "Um Serviço de Persistência de Contexto e Seleção Contextualizada de Documentos para a Arquitetura ClinicSpace" Em: XXXVII Conferência Latinoamericana de Informática (CLEI). Quito, Equador. 2011.

[6] Maran, V., Machado, A., Saccol, D. B., Augustin, I. "A Software Architecture to Provide Persistence and Retrieve of Context Data Based on Ontological Models" Em: IADIS International Conference on WWW/Internet. Rio de Janeiro, Brasil. 2011.

[7] Henricksen, K., Indulska, J., Rakotorirainy, A. "Modeling context information in pervasive computing systems" Em: 1st International Conference on Pervasive Computing, Ed. Springer, pp.167-180. 2002.

[8] Komninos A., Stamou, S. "HealthPal: An Intelligent Personal Medical Assistant for Supporting the Self-Monitoring of Healthcare in the Ageing Society" Em: The 4th International Workshop on Ubiquitous Computing for Pervasive Healthcare Applications. UbiHealth 2006.

[9] Ranganathan, A. "Olympus: a High-Level Programming Model for Pervasive Computing Environments" Em: 3rd IEEE International Conference on Pervasive Computing and Communications. p.716, 2005.

[10] Silva, F. et al "Introduzindo a orientação a tarefas clínicas em um middleware de gerenciamento do espaço pervasivo", Em: II Workshop on Pervasive and Ubiquitous Computing. WPUC. 2008.

[11] Rizzetti, T. A. “Um Ambiente de Contexto Personalizado e Orientado a Tarefas na Arquitetura ClinicSpace", 118f. Dissertação (Mestrado em Computação) - Universidade Federal de Santa Maria, Santa Maria, RS, 2009.

[12] Chen, G., Kotz, D. "Solar: A pervasive-computing infrastructure for context-aware mobile applications" $\quad$ Em: $\quad$ Technical Report TR2002-421. Disponível em: $<$ http://www.cs.dartmouth.edu/ jcrespo/cms_file/SYS_techReport/292/TR2002-421.pdf >. Acesso em: nov. 2011.

[13] Brézillon, P., Borges, M., Pino, J., Pomerol, J. "Context-awareness in group work: three case studies". Em: IFIP International Conference on Decision Support Systems Decision Support in Uncertain and Complex World, pp. 115--124, Monash University. 2004.

[14] Strang, T., Popien, C. "A context modelling survey", Em: Workshop on advanced context modelling reasoning and management as part of Ubicomp, pp 33-40. 2005.

[15] Gruber, T. "What is an Ontology?" Website. Disponível em: <http://www-ksl.stanford.edu/kst/what-isanontology.html>. Acesso em: dez. 2011.

[16] Guarino, N. Formal Ontology and Information Systems. Proceedings of FOIS'98, Trento, Italy, 6-8 June (1998). Amsterdam, IOS Press, pp. 3--15. (http://www.ladseb.pd.cnr.it/infor/Ontology/Papers/F OIS98.pdf)

[17] World Wide Web Consortium "SWRL: A Semantic Web Rule Language Combining OWL and RuleML"Website. Disponível em: <http://www.w3.org/Submission/SWRL/>. Acesso em: nov. 2011.

[18] Bettinia, C., Brdiczkab, O., Henricksen, K., Indulskad, J., Nicklase, D., Ranganathan, A., Riboni, D. "A survey of Context Modelling and Reasoning Technique" Em: Pervasive and Mobile Computing, 6 (2),

Revista Brasileira de Computação Aplicada (ISSN 2176-6649), Passo Fundo, v. 5, n. 1, p. 26-41, abr. 201340 
161-180, 2010.

[19] Chen, H.; Perich, F.; Finin, T. W.; Joshi, A. "Standard Ontology for Ubiquitous and Pervasive Applications" Em: 1st Annual International Conference on Mobile and Ubiquitous Systems, IEEE. 2004.

[20] Zhang, D., Gu T., Wang, X. "Context-aware Smart Home with Semantic Technology CONON" Em: International Journal of Human-friendly Welfare Robotic Systems 6 (4). 12-20, 2005.

[21] Koay, N., Kataria, P., Juric, R., Oberndorf, P., Terstyanszky, G. "Ontological Support for Managing Non-Functional Requirements In Pervasive Healthcare" Em: 42nd Hawaii IEEE International Conference on System Sciences, 2009.

[22] Rautenberg, S.; Todesco, J.; Steil, A.; Gauthier, F. "Uma Metodologia para o Desenvolvimento de Ontologias" Em: Revista Ciências Exatas e Naturais - RECEN - ISSN 2175-5620. Vol. 10 No. 2, 2008

[23] Laerum, H., Fazvaag, A. "Task-oriented evaluation of electronic medical records systems: development and validation of a questionnaire for physicians", Em: BMC Medical Informatics and Decision Making,1. p. 1-16.3, 2004.

[24] Stanford University “The Protégé Ontology Editor and Knowledge Acquisition System" Website. Disponível em: <http://protege.stanford.edu/>. Acesso em: nov. 2011.

[25] American Society for Testing and Materials "Standard Specification for Continuity of Care Record (CCR)"ASTM Standard ASTM E2369 - 05e1 -. DOI: http://dx.doi.org/10.1520/E2369-05E01, 2011

[26] HL7 "Health Level Seven International Page" Website. Disponível em: <http://www.hl7.org/>. Acesso em: mar. 2012.

[27] O'Connor, M.J., Das, A.K. "SQWRL: A Query Language for OWL", Em: Proc. of 6th OWL: Experiences and Directions Workshop (OWLED2009), 2009.

[28] Jess “Jess, the Rule Engine for the JavaTM Platform" Website. Disponível em: $<$ http://www.jessrules.com/>. Acesso em: nov. 2011.

[29] Villalon, P., Figueroa, M., Castro, M., Pérez, A. A Context Ontology for Mobile Environments. In: Workshop on Context, Information and Ontologies - CIAO 2010 Co-located with EKAW 2010 , October 11, 2010, Lisbon, Portugal. 